\title{
Recent Enhancements to the Development of CFD-Based Aeroelastic Reduced-Order Models
}

\author{
Walter A. Silva * \\ NASA Langley Research Center \\ Hampton, Virginia 23681-0001
}

\begin{abstract}
Recent enhancements to the development of CFD-based unsteady aerodynamic and aeroelastic reduced-order models (ROMs) are presented. These enhancements include the simultaneous application of structural modes as CFD input, static aeroelastic analysis using a ROM, and matched-point solutions using a ROM. The simultaneous application of structural modes as CFD input enables the computation of the unsteady aerodynamic state-space matrices with a single CFD execution, independent of the number of structural modes. The responses obtained from a simultaneous excitation of the CFD-based unsteady aerodynamic system are processed using system identification techniques in order to generate an unsteady aerodynamic state-space ROM. Once the unsteady aerodynamic state-space ROM is generated, a method for computing the static aeroelastic response using this unsteady aerodynamic ROM and a state-space model of the structure, is presented. Finally, a method is presented that enables the computation of matchedpoint solutions using a single ROM that is applicable over a range of dynamic pressures and velocities for a given Mach number. These enhancements represent a significant advancement of unsteady aerodynamic and aeroelastic ROM technology.
\end{abstract}

\section{Introduction}

$\mathbf{T}$ HE goal behind the development of reduced-order models (ROMs) for computational aeroelasticity is aimed at addressing two primary challenges: reducing computational cost and enabling the use of CFD information by other disciplines. Development of a ROM entails the creation of a simplified mathematical model that captures the dominant dynamics of the original system. The simplicity of the ROM yields significant improvements in computational efficiency as compared to the original system, thereby addressing the first challenge. This simplified mathematical representation of the original system is, by design, in a mathematical form suitable for use in a multidisciplinary, preliminary design environment. As a result, interconnection of the ROM with other disciplines is possible, thereby addressing the second challenge.

Silva and Bartels ${ }^{1}$ presented the development of linearized, unsteady aerodynamic state-space models for prediction of flutter and aeroelastic response using the parallelized, aeroelastic capability of the CFL3Dv6.0 code. The results presented provided an important validation of the various phases of the ROM development process. The Eigensystem Realization Algorithm (ERA), ${ }^{2}$ which transforms an impulse response into state-space form, was applied for the development of the aerodynamic state-space models. Flut-

*Senior Research Scientist, Aeroelasticity Branch, NASA Langley Research Center, Hampton, Virginia; AIAA Associate Fellow ter results for the AGARD 445.6 Aeroelastic Wing using the CFL3Dv6.0 code were presented as well, including computational costs. Unsteady aerodynamic state-space models were generated and coupled with a state-space model of the structure within a MATLAB/SIMULINK ${ }^{3}$ environment for rapid calculation of aeroelastic responses including flutter. Aeroelastic responses computed directly using the CFL3Dv6.0 code showed excellent comparison with the aeroelastic responses computed using the CFD-based ROM within the MATLAB/SIMULINK environment.

The aerodynamic impulse responses that were used to generate the unsteady aerodynamic ROM for the AGARD wing were computed using CFL3Dv6.0 via the excitation of one mode at a time. The onemode-at-a-time method is referred to as the Original ROM approach. For a four-mode system such as the AGARD wing, these one-mode-at-a-time computations were not very expensive. However, for more realistic cases where the number of modes can be an order of magnitude or more larger, the one-mode-ata-time method becomes impractical. Raveh, ${ }^{4} \mathrm{Kim}^{5}$ and Kim et $\mathrm{al}^{6}$ have proposed methods that enable the simultaneous application of structural modes as CFD input, greatly reducing the cost of identifying the aerodynamic impulse responses from the CFD code. Raveh's method consists, primarily, of using filtered white Gaussian noise as the input and then using different modeling techniques to define an unsteady aerodynamic model (ARMA, state-space). Kim's method 
consists of using simultaneous staggered step inputs, one per mode, and then recovering the individual responses from this simultaneous excitation.

In another paper, ${ }^{7}$ three new types of input functions are introduced that can be used to simultaneously apply the structural modes to the CFD code while enabling the recovery of the individual responses. This new capability will enable the computation of aerodynamic impulse responses for any number of structural modes using a single CFD execution. Reduced-order models generated using the one-mode-at-a-time method are compared to ROMs using the simultaneous excitation inputs.

In the present paper, a new method for computing static aeroelastic solutions using ROMs is presented. Previously, ROMs were generated about a static aeroelastic condition computed using the CFD code. The applicability of the ROM about that condition was limited to a small range of dynamic pressures that would not deviate too far from the static aeroelastic condition. This limitation also applies to the method presented by Kim et al. ${ }^{6}$ The method presented by Raveh $^{4}$ was applied to the AGARD wing. At zero degrees angle of attack and with a symmetric airfoil shape, this wing does not induce a static aeroelastic response. It is not stated in the reference by Raveh ${ }^{4}$ how that method should be applied to a configuration that includes static aeroelastic effects. With this new capability to use the ROM for computing the static aeroelastic solution, there is no need to compute a separate static aeroelastic solution using the CFD code, thereby improving computational efficiency.

Finally, a new method for computing matched-point aeroelastic solutions without re-execution of the CFD code is presented as well. This new matched-point solution method extends the applicability of a single $\mathrm{ROM}$ to include velocity variations in addition to changes in dynamic pressure in order to simulate a true flight condition (Mach number, velocity, and dynamic pressure).

\section{Description of the CFD and System Identification Methods}

The following subsections describe the parallelized, aeroelastic version of the CFL3Dv6.4 code and the system identification methods employed in the development of a ROM.

\section{CFL3Dv6.4 Code}

The computer code used in this study is the CFL3Dv6.4 code, which solves the three-dimensional, thin-layer, Reynolds averaged Navier-Stokes equations with an upwind finite volume formulation. ${ }^{8-10}$ The code uses third-order upwind-biased spatial differencing for the inviscid terms with flux limiting in the presence of shocks. Either flux-difference splitting or flux-vector splitting is available. The flux-difference splitting method of Roe ${ }^{11}$ is employed in the present computations to obtain fluxes at cell faces. There are two types of time discretization available in the code. The first-order backward time differencing is used for steady calculations while the second-order backward time differencing with subiterations is used for static and dynamic aeroelastic calculations. Furthermore, grid sequencing for steady state and multigrid and local pseudo-time stepping for time marching solutions are employed.

One of the important features of the CFL3Dv6.4 code is its capability of solving multiple zone grids with one-to-one connectivity. Spatial accuracy is maintained at zone boundaries, although subiterative updating of boundary information is required. Coarsegrained parallelization using the Message Passing Interface (MPI) protocol can be utilized in multiblock computations by solving one or more blocks per processor. When there are more blocks than processors, optimal performance is achieved by allocating an equal number of grid points to each processor. As a result, the time required for a CFD-based aeroelastic computation can be dramatically reduced.

Because the CFD and computational structural mechanics (CSM) meshes usually do not match at the interface, $\mathrm{CFD} / \mathrm{CSM}$ coupling requires a surface spline interpolation between the two domains. The interpolation of CSM mode shapes to CFD surface grid points is done as a preprocessing step. Modal deflections at all CFD surface grids are first generated. Modal data at these points are then segmented based on the splitting of the flow field blocks. Mode shape displacements located at CFD surface grid points of each segment are used in the integration of the generalized modal forces and in the computation of the deflection of the deformed surface. The final surface deformation at each time step is a linear superposition of all the modal deflections.

\section{System/Observer/Controller Identification Toolbox (SOCIT)}

In structural dynamics, the realization of discretetime state-space models that describe the modal dynamics of a structure has been enabled by the development of algorithms such as the Eigensystem Realization Algorithm (ERA) ${ }^{2}$ and the Observer Kalman Identification (OKID) ${ }^{12}$ Algorithm. These algorithms perform state-space realizations by using the Markov parameters (discrete-time impulse responses) of the systems of interest. These algorithms have been combined into one package known as the System/Observer/Controller Identification Toolbox (SOCIT) ${ }^{13}$ developed at NASA Langley Research Center. 
The first phase of the ROM development process is the identification of the individual impulse responses for each input/output combination. The PULSE algorithm is used to extract these individual input/output impulse responses from simultaneous input/output responses. For a four-input/four-output system, simultaneous application of all four inputs ${ }^{7}$ yields four output responses. The PULSE algorithm is used to extract the individual sixteen (four times four) impulse responses that associate the response in one of the outputs due to one of the inputs. Details of the PULSE algorithm are provided in the references.

For the second phase of the ROM development process, once the individual sixteen impulse responses are available, they are then processed via the Eigensystem Realization Algorithm (ERA) in order to transform the sixteen individual impulse responses into a fourinput/four-output, discrete-time, state-space model. A brief summary of the basis of this algorithm follows.

A finite dimensional, discrete-time, linear, timeinvariant dynamical system has the state-variable equations

$$
\begin{gathered}
x(k+1)=A x(k)+B u(k) \\
y(k)=C x(k)+D u(k)
\end{gathered}
$$

where $\mathrm{x}$ is an $\mathrm{n}$-dimensional state vector, $\mathrm{u}$ an $\mathrm{m}$ dimensional control input, and y a p-dimensional output or measurement vector with $\mathrm{k}$ being the discrete time index. The transition matrix, A ( $\mathrm{n} \times \mathrm{n})$, characterizes the dynamics of the system. The goal of system realization is to generate constant matrices $(\mathrm{A}, \mathrm{B}, \mathrm{C})$ such that the output responses of a given system due to a particular set of inputs is reproduced by the discretetime state-space system described above.

For the system of Eqs. (1) and (2), the time-domain values of the systems discrete-time impulse response are also known as the Markov parameters and are defined as

$$
Y(k)=C A^{k-1} B
$$

with $\mathrm{B}$ an ( $\mathrm{n} \times \mathrm{m})$ matrix and $\mathrm{C}$ a $(\mathrm{p} \times \mathrm{n})$ matrix. The ERA algorithm begins by defining the generalized Hankel matrix consisting of the discrete-time impulse responses for all input/output combinations. The algorithm then uses the singular value decomposition (SVD) to compute the $\mathrm{A}, \mathrm{B}$, and $\mathrm{C}$ matrices.

In this fashion, the ERA is applied to unsteady aerodynamic impulse responses to construct unsteady aerodynamic state-space models.

\section{Static/Dynamic Aeroelastic CFD-Based Analyses}

Example Configuration-Rigid Semi-Span CFD Model

The configuration used for this section of the paper is a supersonic semi-span wind-tunnel model known as the Rigid Semi-span Model (RSM). This configuration has been tested at the NASA Langley's Transonic Dynamics Tunnel (TDT) several times. As the name implies, the wind-tunnel model is fairly rigid. However, as part of collaborative studies between the NASA Langley Research Center and the Boeing Company (Seattle), a "softened" CFL3D model of the RSM was developed by Dr. Moeljo Hong (Boeing). ${ }^{14}$ The model was "softened" by simply reducing the four modal frequencies by a factor of four. Computational results to be presented in this section of the paper are for Euler (inviscid)solutions at a Mach number of 0.7 and an angle of attack of 3 degrees. This configuration does not have a symmetric airfoil and will, therefore, at practically all angles of attack, generate a static aeroelastic response in addition to the dynamic aeroelastic response. Figure 1 presents the surface grid for the CFL3D RSM configuration.

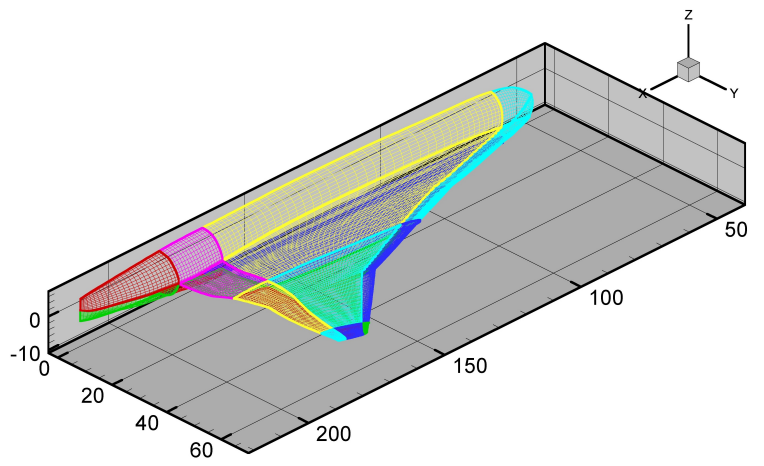

Fig. 1 Computational grid of supersonic semi-span configuration.

\section{Traditional Approach}

The traditional process for generating CFD-based aeroelastic responses consists of three steps: 1). perform computation of a converged steady, rigid solution at a given Mach number and angle of attack; 2). at the same Mach number and angle of attack, perform computation of a converged static aeroelastic solution at a selected dynamic pressure and velocity; 3 ). at the same Mach number, angle of attack, dynamic pressure, and velocity, perform computation of a dynamic aeroelastic response. A sketch depicting this process is presented as Figure 2. This method was applied successfully by Silva and Bennett ${ }^{15}$ for prediction of the aeroelastic responses of the Active Flexible Wing (AFW) wind-tunnel model. 


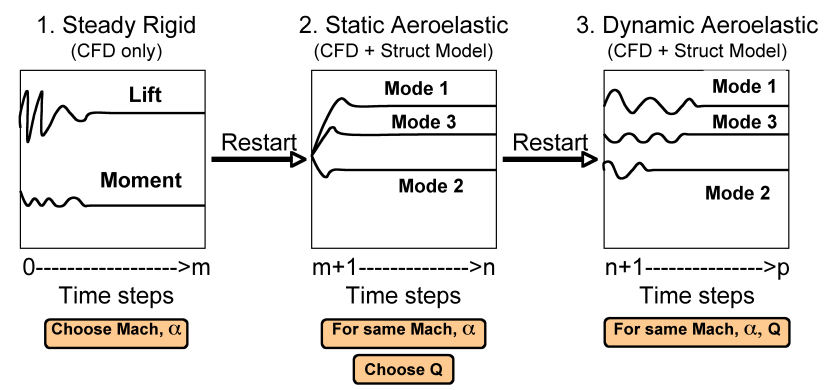

Fig. 2 Sketch depicting the traditional process for generating CFD-based aeroelastic responses.

The static aeroelastic solution is computed by restarting the converged steady, rigid solution. The converged steady, rigid solution, therefore serves as the initial condition for the static aeroelastic solution. Likewise, the converged static aeroelastic solution serves as the initial condition for the dynamic aeroelastic solution. The static aeroelastic solution is computed by imposing a very large value of modal damping to the system, thereby attenuating dynamic transients and yielding static aeroelastic deflections. In addition, the initial conditions (initial generalized displacements and velocities) are set to zero within the structural integrator portion of CFL3D. An example of a static aeroelastic solution for the softened RSM configuration is presented as Figure 3 where the artificially-excessive modal damping (0.99) results in an acceleration of the static aeroelastic convergence by attenuating dynamic transients.

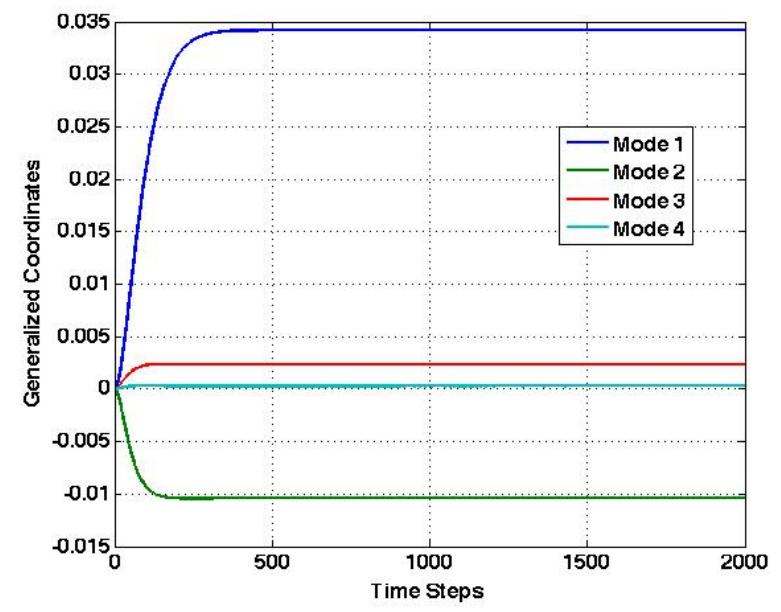

Fig. 3 Converged static aeroelastic solution for the RSM configuration at 0.7 Mach number, 3 degrees angle of attack, and 0.1 psi dynamic pressure.

Upon achieving a converged static aeroelastic solution, a dynamic solution is computed by restarting the static aeroelastic solution. The restarting of a solution file basically defines the initial structural and flow conditions from the previous solution (the static aeroelastic solution in this case). For the dynamic solution, the value of modal damping is set to a realistic value (typically zero or on the order of 1-3 percent of critical). The initial conditions of the structure are now set to non-zero values in order to provide an initial excitation to the system. Typically, generalized velocities are set to some small value while the generalized displacements are set to zero. The resultant dynamic aeroelastic solution for zero modal damping and a value of 0.001 for all four generalized velocities is presented as Figure 4. Zooming in on the first generalized coordinate, presented as Figure 5, the stability of the dynamic response can be ascertained. However, post-processing of the generalized aerodynamic transients is required to obtain damping and frequency estimates.

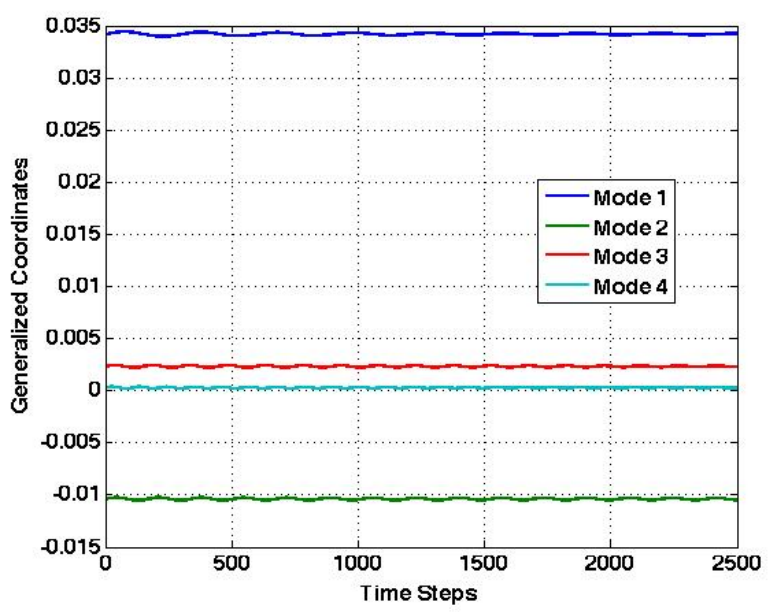

Fig. 4 Dynamic aeroelastic response for the RSM configuration at 0.7 Mach number, 3 degrees angle of attack, and 0.1 psi dynamic pressure about the converged static aeroelastic response at the same conditions.

The primary reason for performing separate and serial static and dynamic aeroelastic solutions ${ }^{15}$ was for computational efficiency, as follows. Clearly, a solution can be obtained which contains both the static and dynamic solutions occurring simultaneously by setting modal damping to zero (or a small value), setting the structural initial conditions (generalized velocities) to small non-zero values, and restarting this combined solution from the restart file of a converged steady, rigid solution. A combined solution for a dynamic pressure of 0.1 psi is presented as Figure 6 .

For some configurations, when performing a combined solution, the convergence of the static portion of the response could require a large number of time steps, making it difficult to clearly define the dynamic portion of the response (stability). Therefore, in sepa- 


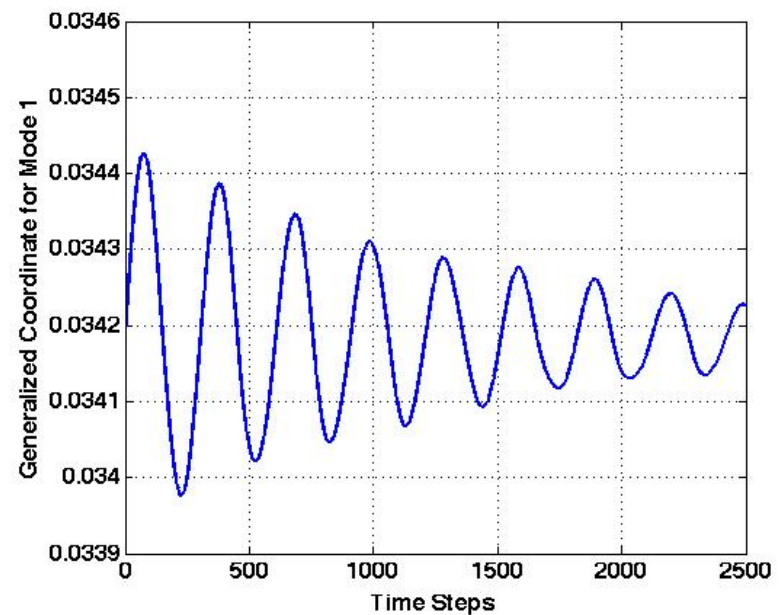

Fig. 5 Blow up of the first mode dynamic aeroelastic response from Figure 4.

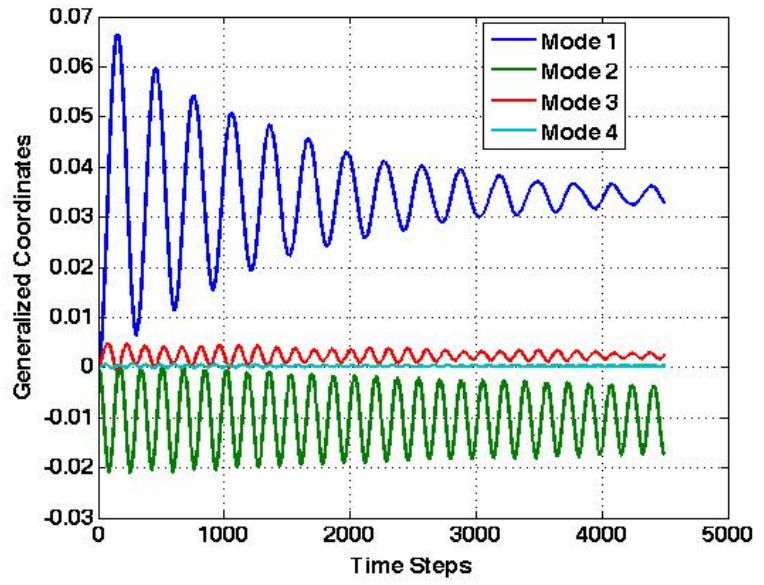

Fig. 6 Combined CFL3D aeroelastic solution that includes static and dynamic solutions simultaneously for the RSM configuration at 0.7 Mach number, 3 degrees angle of attack, and 0.1 psi dynamic pressure.

rating the solution process and artificially accelerating the static aeroelastic solution, that portion of the computational cost can be reduced. Then, the dynamic solution can be generated without the added effect of a static variation and, subsequently, may be easier to interpret. However, this process may need to be revisited for complex configurations to determine if, indeed, a significant computational cost savings is always achieved. In some cases, it is quite feasible that a combined solution may require fewer time steps than the individual time steps required for separate static and dynamic solutions. One possible strategy may be to initially perform a combined solution at a given dynamic pressure in order to get a sense of the static and dynamic properties of the configuration of interest. Based on that result, one might be able to make better decisions regarding the computational costs associated with performing separate static and dynamic solutions.

\section{Previous ROM Approach}

To date, the system-identification, time-domainbased methods for generating unsteady aerodynamic ROMs have relied on the traditional approach of separate and serial static and dynamic aeroelastic solutions presented above. For some methods, ${ }^{1,4}$ the configuration investigated did not involve any static aeroelastic deformation. This is true for the AGARD 445.6 Wing which has a symmetric airfoil shape and, at zero degrees angle of attack, does not induce any static aeroelastic deformation. For other methods, ${ }^{6}$ where the configuration induced a static aeroelastic effect, the unsteady aerodynamic ROM was generated about a converged static aeroelastic solution. That is, the ROM was generated using the initial conditions (restart) resulting from the converged static aeroelastic solution. This implies that the ROM was generated at the dynamic pressure at which the static aeroelastic solution was computed. Variation of the dynamic pressure via the ROM (which is how the ROM is used to explore the dynamic pressure solution space) then implies ROM solutions that are removed from the static aeroelastic condition about which the ROM was originally generated. It is reasonable to expect that the accuracy of the ROM generated in this fashion will diminish the greater the difference between the dynamic pressure at which the ROM was generated and the dynamic pressure at which a ROM solution is desired.

\section{Enhanced ROM Approach}

For realistic configurations, the issue of static aeroelastic effects must be included in any ROM development. Because the accepted process used to generate ROMs was based on generating the ROM about a converged static aeroelastic solution, it was assumed that ROMs could not be used to compute static aeroelastic solutions. To date, no method had been identified for using a ROM for computing static aeroelastic solutions.

Careful analysis of the traditional approach for computing the separate and serial static and dynamic aeroelastic solutions indicates that the only parameters that vary from one solution (the static solution) to the next (the dynamic solution) are associated with the structure. That is, to compute a static aeroelastic solution, the modal damping is set to a very high value (0.99) and the structural initial conditions are set to zero. Then, to compute the dynamic aeroelastic response (restarted from the static aeroelastic solution), the modal damping is set to zero (or a 
small value) and the structural initial conditions (generalized velocities) are set to a small non-zero value. Therefore, if only a variation in structural parameters differentiate a static from a dynamic solution, the unsteady aerodynamic system used for both solutions is clearly identical. Following that thought, then, if an unsteady aerodynamic ROM can be used to predict dynamic aeroelastic responses (which comprises all of the system-identification, time-domain-based ROM results to date), surely that same unsteady aerodynamic ROM can be used to predict static aeroelastic responses. The first conclusion, then, is that the unsteady aerodynamic ROM should not be generated about a static aeroelastic solution as that negates the whole point of using unsteady aerodynamic ROMs for computing static aeroelastic solutions. The unsteady aerodynamic ROM must therefore be generated from the steady, rigid solution.

The next step is to understand how a static aeroelastic effect is induced within the CFL3D code (or any aeroelastic CFD code) and how that effect can be simulated using an unsteady aerodynamic ROM. The answer becomes clear when we consider two airfoils: a symmetric airfoil at zero degrees angle of attack and a nonsymmetric airfoil at any angle of attack. For a symmetric airfoil at zero degrees angle of attack, the pressures on the lower surface are identical to the pressures on the upper surface resulting in a zero net pressure difference. Since it is the pressure difference that is integrated with each mode shape to yield the generalized forces, the symmetric airfoil at zero degrees angle of attack, without any structural perturbation, does not generate any initial generalized forces. On the other hand, the nonsymmetric airfoil will induce a non-zero pressure difference which is then integrated with each mode shape to yield non-zero, initial generalized aerodynamic forces (GAFs). Therefore, it is this aerodynamic initial condition, consisting of non-zero GAFs, that is responsible for initiating (and perpetuating) a static aeroelastic response.

Figure 7 contains a schematic of the SIMULINK system used for predicting dynamic aeroelastic responses. The unsteady aerodynamic ROM (shown as the Discrete State-Space Model of Aerodynamics) was generated in one of two ways: 1). about a steady, rigid solution for a configuration without static aeroelastic effects or 2). about a converged static aeroelastic solution for a configuration with static aeroelastic effects.

In order to simulate static aeroelastic effects, the initial GAFs (induced by non-zero pressure difference on wing) are treated as a bias that is added to the unsteady aerodynamic ROM. The resultant SIMULINK model is presented as Figure 8. Note that the unsteady aerodynamic ROM shown in Figure 8 was generated about the steady, rigid solution. The initial values of
GAFs shown in the stacked boxes in Figure 8 were obtained from the first time step of an aeroelastic solution at a selected dynamic pressure. By selecting these values from the solution at a given dynamic pressure, there still exists some limitation of how far the ROM can be exercised in terms of dynamic pressure. However, generating these initial GAFs at different dynamic pressures is computationally inexpensive. There is a computational cost savings from not having to execute a full static aeroelastic solution.

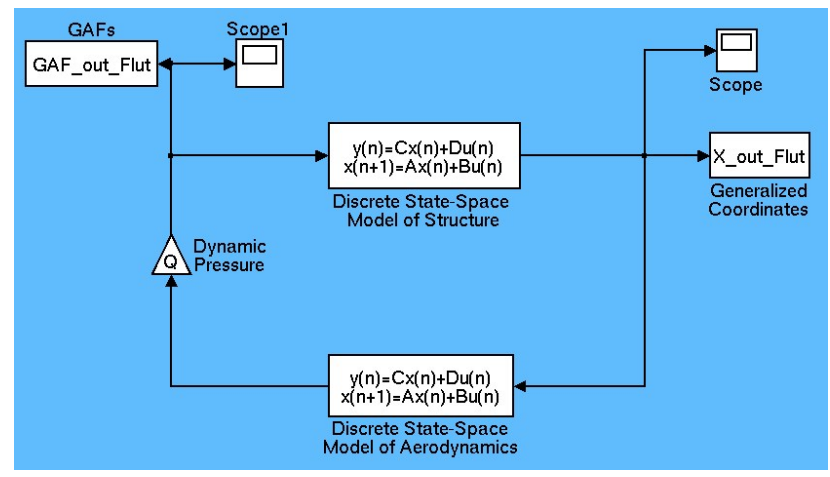

Fig. 7 SIMULINK model used for predicting dynamic aeroelastic responses only.

Another important point to make is with regards to the level of excitation used to generate an unsteady aerodynamic ROM. In the previous method, where a ROM is generated about a converged static aeroelastic solution (i.e., a particular dynamic pressure), the selection of the dynamic pressure defines a region of aeroelastic behavior that is of interest. For example, if it is expected that the unsteady flow field will vary significantly beyond some elastic deformation of the structure, that elastic deformation corresponds to some value of dynamic pressure. The desired ROM can then be generated about that condition in order to capture important unsteady aerodynamic effects. With the new method for generating ROMs, since the unsteady aerodynamic ROM is generated from a steady, rigid solution, it is independent of dynamic pressure. With the new method, instead of using dynamic pressure to excite a particular range of unsteady aerodynamic behavior, it is the magnitude of the generalized coordinates (used as input to the unsteady aerodynamic system to define the ROM) that defines a particular region of interest.

Comparison of various CFL3D and ROM static, dynamic, and combined aeroelastic responses are now presented. In another paper, ${ }^{7}$ three new orthogonal functions are introduced that can be used to simultaneously excite all the modes of an unsteady aerodynamic system in order to generate an unsteady aerodynamic ROM with a single CFD execution. For the results that follow, the unsteady aerodynamic ROM 


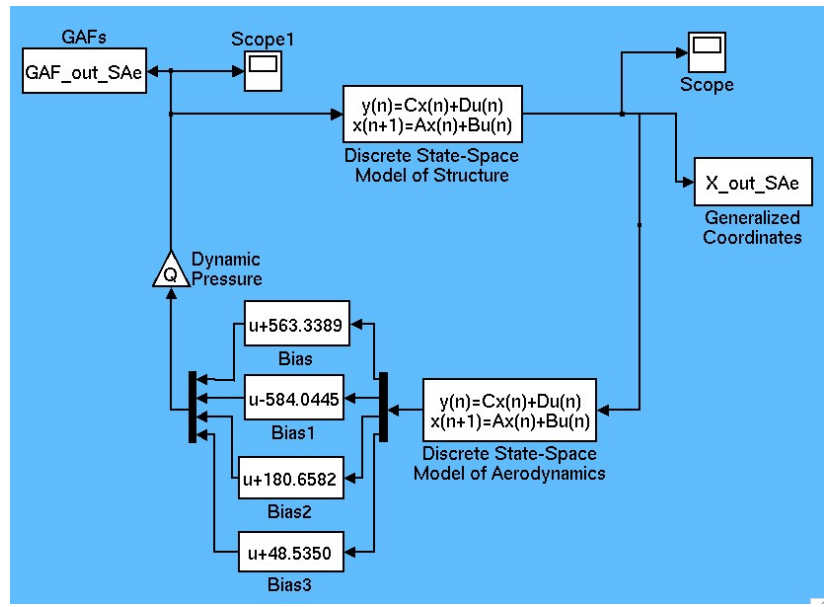

Fig. 8 SIMULINK model used for predicting both or either static and dynamic aeroelastic responses.

was generated using the Walsh functions. ${ }^{7}$

The computation of static aeroelastic results using the ROM requires the modification of the modal damping within the state-space model of the structure presented in Figure 8. The modal damping for the structural state-space model is set to 0.99 and the initial conditions (also of the structure) are set to zero. A comparison of the static aeroelastic results computed using CFL3D already presented in Figure 3 and those computed using the ROM at a dynamic pressure of 0.1 psi is presented in Figure 9. The results compare very well, with a slight mismatch of the static aeroelastic response for the first generalized coordinate. This mismatch may be due to the selection of various parameters within the system identification process used to generate the ROM.

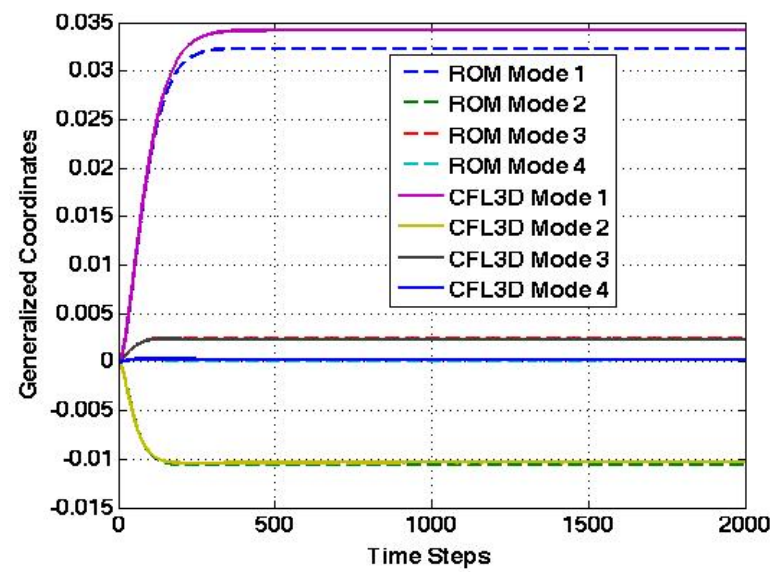

Fig. 9 Comparison of CFL3D and ROM static aeroelastic responses for the RSM configuration at 0.7 Mach number, 3 degrees angle of attack, and 0.1 psi dynamic pressure.

The same ROM configuration presented in Figure 8 can now be used to compute a combined static and dynamic aeroelastic solution at the same dynamic pressure. The modal damping of the structure is now set to zero and the initial conditions are set to the same values used in the CFL3D solution (generalized velocities equal to 0.001). Then the ROM solution is computed and the result is presented in Figure 10.

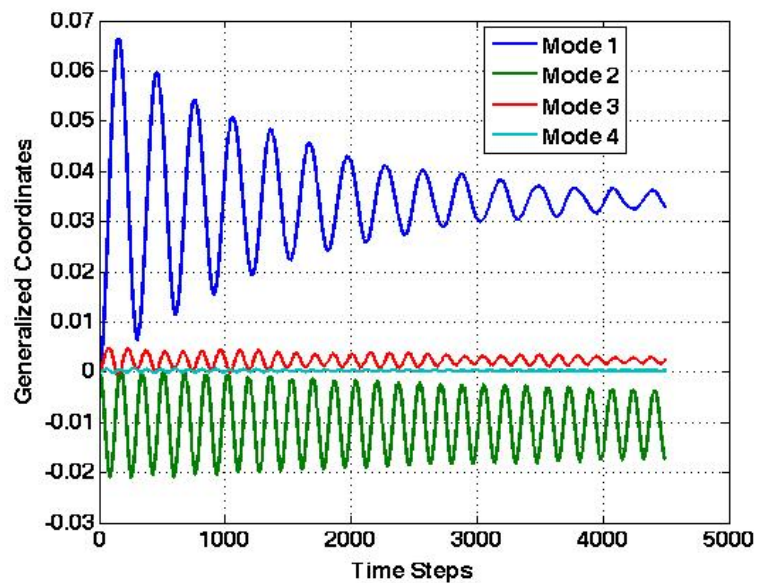

Fig. 10 Combined ROM static and dynamic aeroelastic solutions for the RSM configuration at 0.7 Mach number, 3 degrees angle of attack, and 0.1 psi dynamic pressure.

It can be seen that the comparison between the CFL3D (Figure 6) and ROM combined (static and dynamic) aeroelastic solutions is very good. The comparison of these responses for the first mode is presented as Figure 11.

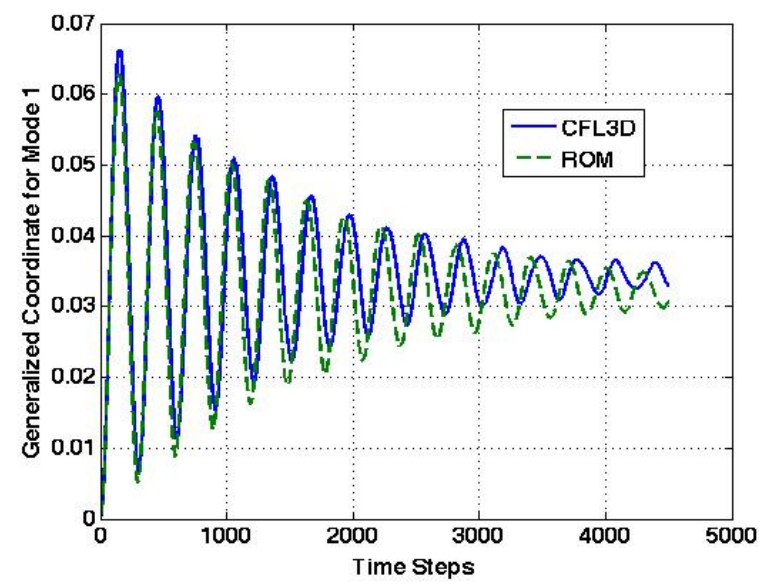

Fig. 11 Comparison of CFL3D and ROM combined aeroelastic response for the first mode for the RSM configuration at 0.7 Mach number, 3 degrees angle of attack, and 0.1 psi dynamic pressure.

The comparison of combined solutions for CFL3D and ROM for a dynamic pressure of $0.5 \mathrm{psi}$ is presented 
in Figure 12. A discrepancy is noticed with respect to the static aeroelastic values and increased damping for the ROM solutions. Again, this discrepancy may be due to the particular set of parameters used within the system identification techniques to generate the ROM.

A comparison of only the dynamic portions of these responses is presented in Figure 13. This comparison has benefitted from the removal of the static aeroelatic effect. However, additional analyses are required to fully understand the source of some of these discrepancies.

Finally, a comparison of CFL3D and ROM dynamic aeroelastic responses at a dynamic pressure of 0.9 psi is presented in Figure 14. Although there exist some differences between some of the generalized coordinate responses, both the CFL3D and ROM solutions are at or near flutter.

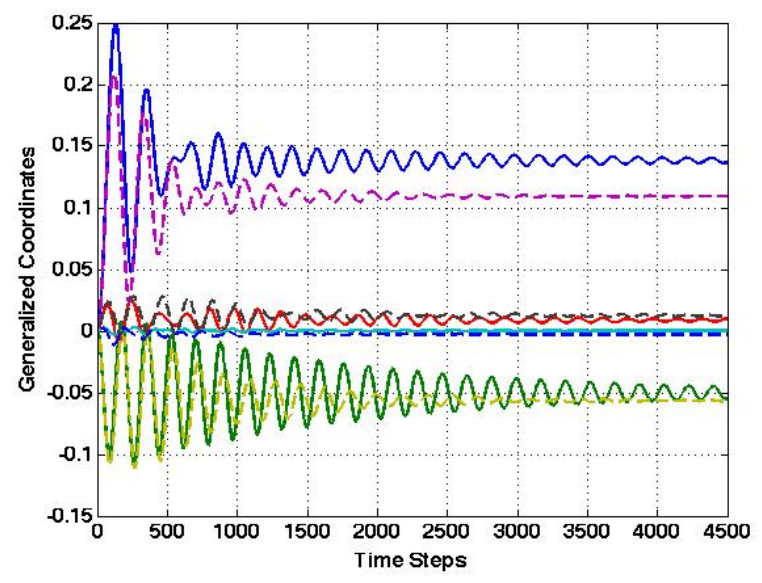

Fig. 12 Comparison of CFL3D (solid lines) and ROM (dashed lines) combined aeroelastic responses for the RSM configuration at 0.7 Mach number, 3 degrees angle of attack, and 0.5 psi dynamic pressure.

This enhanced method shows promise towards the use of ROMs for full static and dynamic aeroelastic responses required for realistic configurations. In addition, subsequent research will investigate the effects of the different input functions (used to generate a ROM), input amplitudes, record length, and various SOCIT processing options on the aeroelastic responses using unsteady aerodynamic ROMs.

\section{Matched-Point CFD-Based Analyses}

Example Configuration-AGARD 445.6 Aeroelastic Wing

For the discussion regarding the ROM-based matched-point solution method, a CFL3D model of the AGARD 445.6 Aeroelastic Wing is used. The AGARD 445.6 Aeroelastic Wing has been used extensively by several authors to validate computational

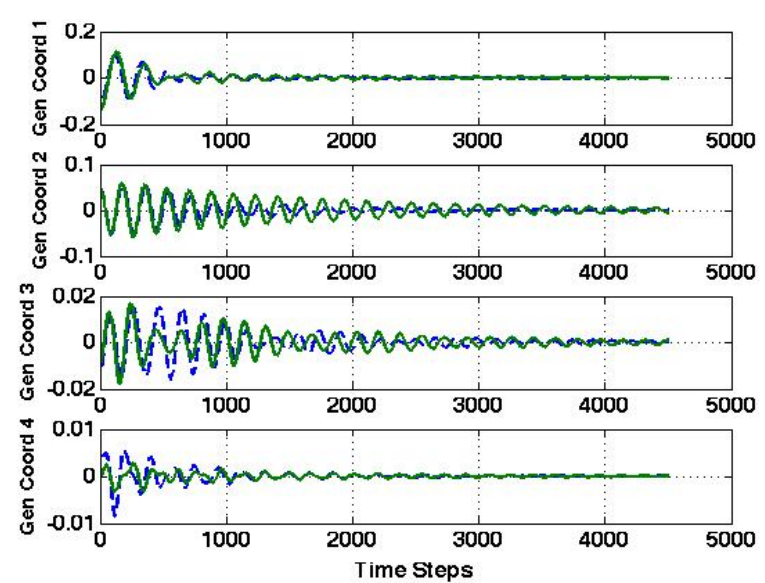

Fig. 13 Comparison of CFL3D (solid green line) and ROM (dashed blue line) dynamic aeroelastic responses for all four generalized coordinates for the RSM configuration at 0.7 Mach number, 3 degrees angle of attack, and 0.5 psi dynamic pressure.

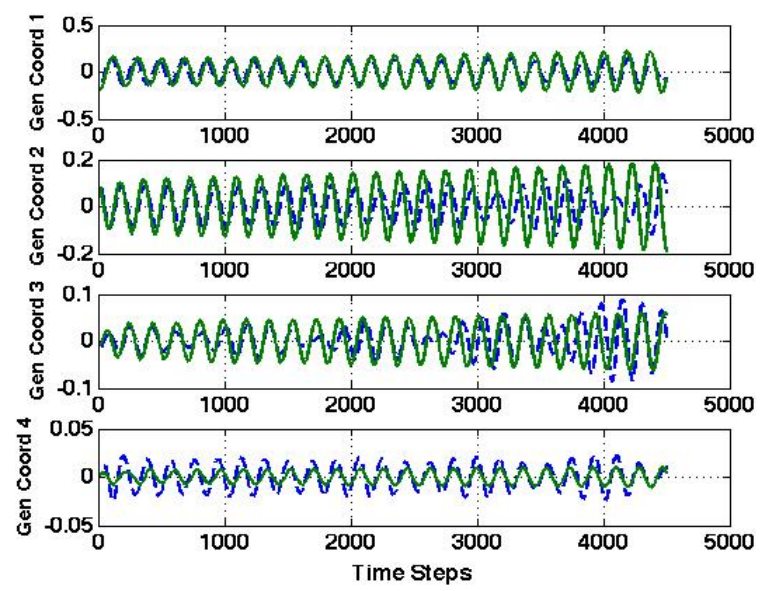

Fig. 14 Comparison of CFL3D (solid green line) and ROM (dashed blue line) dynamic aeroelastic responses for all four generalized coordinates for the RSM configuration at 0.7 Mach number, 3 degrees angle of attack, and 0.9 psi dynamic pressure.

methods. ${ }^{16-18}$ Although the aeroelastic behavior of this wing is fairly benign (weakly nonlinear), the aeroelastic data from the flutter test of this wing provides a good starting point for validation of computational techniques. $^{19}$ The wing is a 45 -degree swept-back wing with a NACA $65 \mathrm{~A} 004$ airfoil section, panel aspect ratio of 1.65, and a taper ratio of 0.6576. Results presented are for Euler solutions using the grid presented as Figure 15. Additional details regarding this wing can be found in the references. 


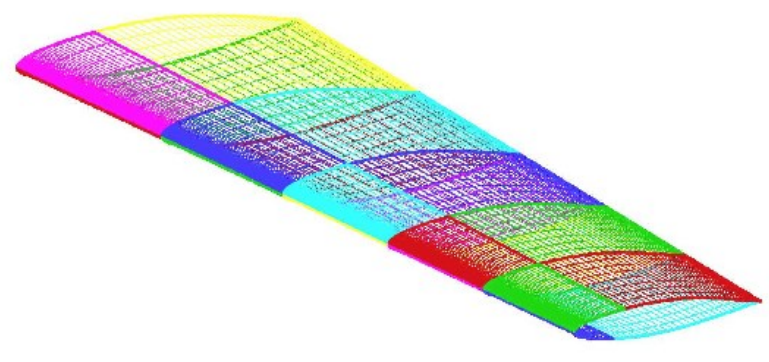

Fig. 15 CFL3D grid for AGARD 445.6 Aeroelastic Wing.

\section{Traditional Approach}

Another recent enhancement to the ROM methodology involves the use of a single ROM to compute matched-point solutions. Typically, for realistic configurations that fly in the atmosphere, it is important to understand the aeroelastic response of that vehicle at the corresponding atmospheric conditions. That is, a given altitude and Mach number define a particular dynamic pressure. This is referred to as a matchedpoint solution.

When performing aeroelastic CFD analyses, a matched-point solution is obtained by setting the Mach number, velocity, and dynamic pressure of interest. These three parameters together define a particular flight condition. As discussed above, an unsteady aerodynamic ROM is defined based on dynamic pressure variations; changes in dynamic pressure is also how the ROM is typically used for predicting aeroelastic responses. If a different matched-point solution is desired, this requires a re-execution of the CFD code at the new conditions (Mach number, dynamic pressure, and velocity). But a ROM, as discussed above, only captures the effect of dynamic pressure variations since, during the identification process, the velocity is kept constant. Different matched-point solutions would imply the need for different ROMs for the different conditions.

\section{Enhanced ROM Approach}

In order to minimize computational cost, it would be beneficial if a given unsteady aerodynamic ROM could be used to predict aeroelastic responses at any dynamic pressure and velocity. This issue involves understanding how velocity enters the aeroelastic equa- tions of motion within an aeroelastic CFD solver.

The answer to this issue lies in the discretization of the equations, in particular, the definition of the time step used in the CFD solution. The equation for the actual (dimensional) time step is

$$
d t a c t=\{d t * \text { Machnumber } * \text { rlength/velocity }\}
$$

with dt being the non-dimensional time step and rlength is a reference length. Therefore, within an aeroelastic CFD solver, the effect of velocity is to alter the time step of numerical integration. With this in mind, a matched-point solution can be realized using a single unsteady aerodynamic ROM by simply modifying the sampling rate of the discrete state-space models shown in Figure 8 (aerodynamic and structural) such that the new sampling rate corresponds to the time step associated with a particular velocity back in the CFD code. Kim et al ${ }^{6}$ introduce a similar concept but the present discretization is directly connected to the CFD time step (as defined within CFL3Dv6.4). This is a very simple concept and the results are presented below.

Figure 16 presents a comparison of the generalized coordinate responses generated using a single unsteady aerodynamic ROM of the AGARD 445.6 $\mathrm{Wing}^{1}$ at a dynamic pressure of $75 \mathrm{psf}$ and a velocity of 973 $\mathrm{ft} / \mathrm{sec}$ and the corresponding generalized coordinate responses from the direct CFL3D solution, both at $\mathrm{M}=0.9$. These results were computed using the onemode-at-a-time approach. Clearly, since the ROM used for this analysis was generated at this velocity, the comparison is excellent.

As stated above, the goal of the ROM matchedpoint solution technique is to be able to use a single unsteady aerodynamic ROM to predict the response of the aeroelastic system at various combinations of dynamic pressure and velocity in order to generate matched-point solutions. Defining a new time step based on a different velocity, a modified unsteady aerodynamic ROM and structural state-space model are generated using re-sampling techniques available in MATLAB. This is done by basically resampling the discrete-time systems at the new time step. Figure 17 presents a comparison of the generalized coordinate responses generated using a re-sampled version of the unsteady aerodynamic ROM at a dynamic pressure of $70 \mathrm{psf}$ and a velocity of $400 \mathrm{ft} / \mathrm{sec}$ and the corresponding generalized coordinate responses from the direct CFL3D solution. As can be seen, the matched-point technique enables the use of a single unsteady aerodynamic ROM (although re-sampled to match velocity) to compute the response of the aeroelastic system to a variation in dynamic pressure and velocity.

Likewise, Figure 18 presents a comparison of the

9 OF 11 
generalized coordinate responses generated at a dynamic pressure of $80 \mathrm{psf}$ and a velocity of 1000 $\mathrm{ft} / \mathrm{sec}$ and the corresponding generalized coordinate responses from the direct CFL3D solution. Once again, the comparison is excellent and serves to validate the newly-developed matched-point ROM solution.
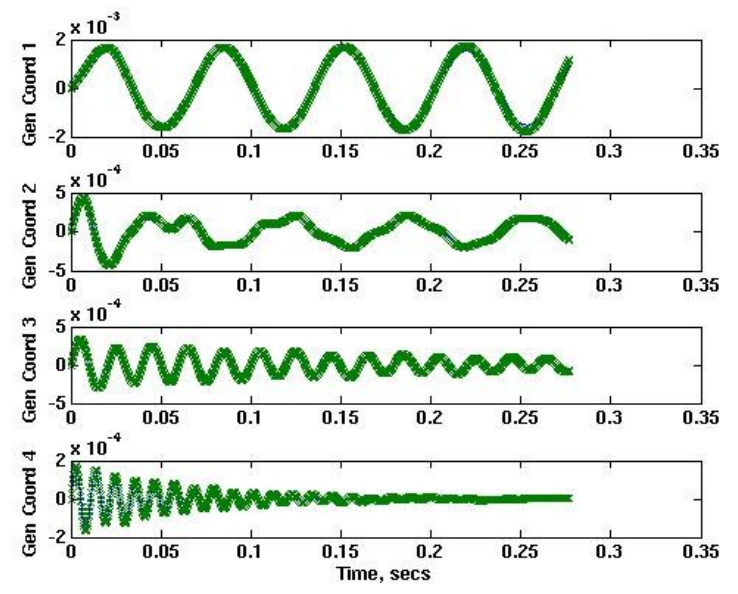

Fig. 16 Comparison of generalized coordinates from the ROM and the full CFL3D solution for the AGARD wing at $\mathrm{M}=0.9, \mathrm{Q}=75 \mathrm{psf}$ and $\mathrm{U}=973$ $\mathrm{ft} / \mathrm{sec}$.
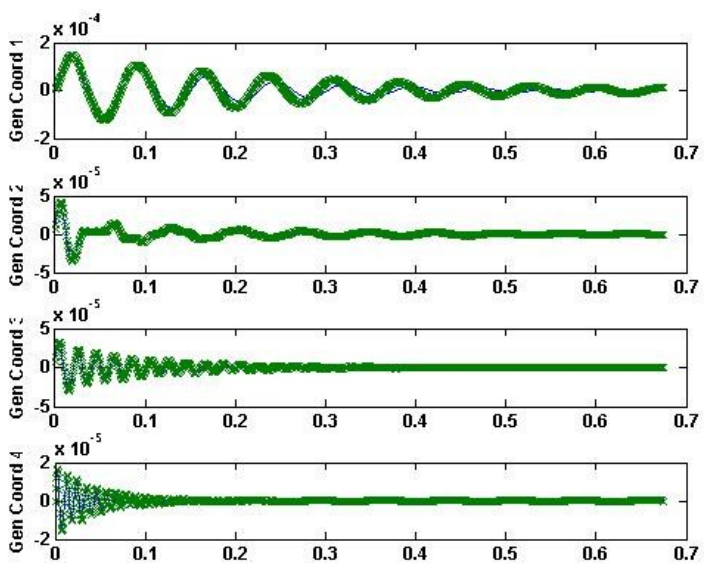

Fig. 17 Comparison of generalized coordinates from the ROM and the full CFL3D solution for the AGARD wing at $\mathrm{M}=\mathbf{0 . 9}, \mathrm{Q}=\mathbf{7 0} \mathrm{psf}$ and $\mathrm{U}=400$ $\mathrm{ft} / \mathrm{sec}$.

\section{Concluding Remarks}

Recent enhancements to the development of aeroelastic reduced-order models (ROMs) have been presented. These enhancements include the capability to compute combined static and dynamic aeroelastic responses and matched-point solutions using a single
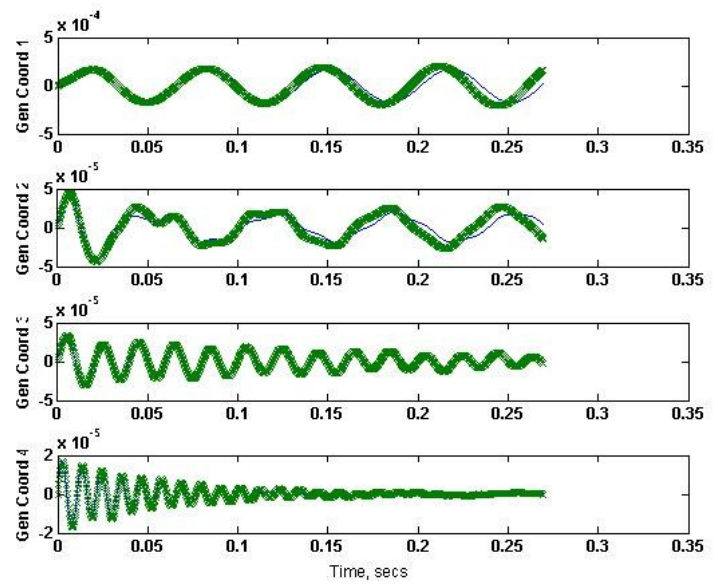

Fig. 18 Comparison of generalized coordinates from the ROM and the full CFL3D solution for the AGARD wing at $\mathrm{M}=\mathbf{0 . 9}, \mathrm{Q}=80 \mathrm{psf}$ and $\mathrm{U}=1000$ $\mathrm{ft} / \mathrm{sec}$.

ROM. The simultaneous application of the structural modes as input to the CFD was briefly described as the details of this enhancement were provided in a separate paper. The ability to compute static and dynamic aeroelastic responses using the ROM was presented. Combined static and dynamic aeroelastic responses were computed using a ROM of the RSM supersonic configuration. These combined responses were compared with similar responses from the CFL3D code. The comparisons indicated reasonable correlation, depending on the dynamic pressure of interest. Additional research is underway to identify the source of some discrepancies as well as to optimize the overall process. Finally, the matched-point solution enhancement was shown to accurately compute aeroelastic responses of a given ROM of the AGARD 445.6 wing at various dynamic pressures and velocities. These new enhancements to the development of aeroelastic ROMs provides a significant advancement in ROM technology and enables the practical and efficient application of ROM technology to real-world problems.

\section{References}

${ }^{1}$ Silva, W. A. and Bartels, R. E., "Development of ReducedOrder Models for Aeroelastic Analysis and Flutter Prediction Using the CFL3Dv6.0 Code," Journal of Fluids and Structures, No. 19, 2004, pp. 729-745.

${ }^{2}$ Juang, J.-N. and Pappa, R. S., "An Eigensystem Realization Algorithm for Modal Parameter Identification and Model Reduction," Journal of Guidance, Control, and Dynamics, Vol. 8, 1985, pp. 620-627.

3 "Registered Product of the MathWorks, Inc." .

${ }^{4}$ Raveh, D. E., "Identification of Computational-FluidDynamic Based Unsteady Aerodynamic Models for Aeroelastic Analysis," Journal of Aircraft, Vol. 41, June 2004, pp. 620-632.

${ }^{5} \mathrm{Kim}, \mathrm{T}$., "Efficient Reduced-Order System Identification for Linear Systems with Multiple Inputs," AIAA Journal, Vol. 43, 2005, pp. 1455-1464. 
${ }^{6}$ Kim, T., Hong, M., Bhatia, K. G., and SenGupta, G., "Aeroelastic Model Reduction for Affordable Computational Fluid Dynamics-Based Flutter Analysis," AIAA Journal, Vol. 43, 2005, pp. 2487-2495.

${ }^{7}$ Silva, W. A., "Simultaneous Excitation of Multiple-Input Multiple-Output CFD-Based Unsteady Aerodynamic Systems," 48th AIAA/ASME/ASCE/AHS/ASC Structures, Structural Dynamics, and Materials Conference, No. AIAA Paper No. 2007-1988, Honolulu, HI, April 23-26 2007.

${ }^{8}$ Krist, S. L., Biedron, R. T., and Rumsey, C. L., "CFL3D User's Manual Version 5.0," Tech. rep., NASA Langley Research Center, 1997.

${ }^{9}$ Bartels, R. E., "Mesh Strategies for Accurate Computations of Unsteady Spoiler and Aeroelastic Problems," AIAA Journal of Aircraft, Vol. 37, 2000, pp. 521-525.

${ }^{10}$ Bartels, R. E., Rumsey, C. L., and Biedron, R. T., "CFL3D Version 6.4: General Usage and Aeroelastic Analysis," NASA TM 2006 214301, April 2006.

${ }^{11}$ Roe, P. L., "Approximate Riemann Solvers, Parameter Vectors, and Difference Schemes," Journal of Computational Physics, Vol. 43, 1981, pp. 357-372.

${ }^{12}$ Juang, J.-N., Phan, M., Horta, L. G., and Longman, R. W., "Identification of Observer/Kalman Filter Markov Parameters: Theory and Experiments," Journal of Guidance, Control, and Dynamics, Vol. 16, 1993, pp. 320-329.

${ }^{13}$ Juang, J.-N., Applied System Identification, Prentice-Hall PTR, 1994.

${ }^{14}$ Hong, M., Kuruvila, G., Bhatia, K., SenGupta, G., and Kim, T., "Evaluation of CFL3D for Unsteady Pressure and Flutter Predictions," 44th AIAA/ASME/ASCE/AHS/ASC Structures, Structural Dynamics, and Materials Conference, No. AIAA Paper No. 2003-1923, Norfolk, VA, April 7-10 2003.

${ }^{15}$ Silva, W. A. and Bennett, R. M., "Application of Transonic Small Disturbance Theory to the Active Flexible Wing Model," Journal of Aircraft, Vol. 32, 1995, pp. 16-22.

${ }^{16}$ Gordnier, R. E. and Melville, R. B., "Transonic Flutter Simulations Using an Implicit Aeroelastic Solver," AIAA Journal of Aircraft, Vol. 37, 2000, pp. 872-879.

${ }^{17}$ Gupta, K. K., Voelker, L. S., Bach, C., Doyle, T., and Hahn, E., "CFD-Based Aeroelastic Analysis of the X-43 Hypersonic Flight Vehicle," Proceedings of the 39th Aerospace Sciences Meeting and Exhibit, No. 2001-0712, Reno, CA, Jan. 2001.

${ }^{18}$ Lee-Rausch, E. M. and Batina, J. T., "Wing Flutter Computations Using an Aerodynamic Model Based on the NavierStokes Equations," Journal of Aircraft, Vol. 33, 1993, pp. 11391148 .

${ }^{19}$ E. C. Yates, J., Land, N. S., and J. T. Foughner, J., "Measured and Calculated Subsonic and Transonic Flutter Characteristics of a 45-degree Swept-Back Wing Planform in Air and in Freon-12 in the Langley Transonic Dynamics Tunnel," Tech. rep., NASA, TN D-1616, 1963. 